\title{
Noninvasive vagus nerve stimulation as acute therapy for migraine
}

\author{
The randomized PRESTO study
}

Cristina Tassorelli, MD, PhD, Licia Grazzi, MD, Marina de Tommaso, MD, PhD, Giulia Pierangeli, MD, PhD, Paolo Martelletti, MD, PhD, Innocenzo Rainero, MD, PhD, Stefanie Dorlas, BMath, BEd,

Pierangelo Geppetti, MD, PhD, Anna Ambrosini, MD, PhD, Paola Sarchielli, MD, PhD, Eric Liebler, and Piero Barbanti, MD, PhD, On behalf of the PRESTO Study Group

Neurology ${ }^{\circledR}$ 2018;91:e364-e373. doi:10.1212/WNL.0000000000005857

\section{Abstract}

\section{Objective}

To evaluate the efficacy, safety, and tolerability of noninvasive vagus nerve stimulation (nVNS; gammaCore; electroCore, LLC, Basking Ridge, NJ) for the acute treatment of migraine in a multicenter, double-blind, randomized, sham-controlled trial.

\section{Methods}

A total of 248 participants with episodic migraine with/without aura were randomized to receive $n V N S$ or sham within 20 minutes from pain onset. Participants were to repeat treatment if pain had not improved in 15 minutes.

\section{Results}

nVNS $(\mathrm{n}=120)$ was superior to sham $(\mathrm{n}=123)$ for pain freedom at 30 minutes $(12.7 \%$ vs $4.2 \%$; $p=0.012)$ and 60 minutes $(21.0 \%$ vs $10.0 \%$; $p=0.023)$ but not at 120 minutes $(30.4 \%$ vs $19.7 \%$; $p=0.067$; primary endpoint; logistic regression) after the first treated attack. A post hoc repeatedmeasures test provided further insight into the therapeutic benefit of nVNS through 30,60, and 120 minutes (odds ratio 2.3; 95\% confidence interval 1.2, 4.4; $p=0.012$ ). nVNS demonstrated benefits across other endpoints including pain relief at 120 minutes and was safe and well-tolerated.

\section{Conclusion}

This randomized sham-controlled trial supports the abortive efficacy of nVNS as early as 30 minutes and up to 60 minutes after an attack. Findings also suggest effective pain relief, tolerability, and practicality of nVNS for the acute treatment of episodic migraine.

\section{ClinicalTrials.gov identifier}

NCT02686034.

\section{Classification of evidence}

This study provides Class I evidence that for patients with an episodic migraine, nVNS significantly increases the probability of having mild pain or being pain-free 2 hours poststimulation (absolute difference 13.2\%).

\author{
Correspondence \\ Prof. Tassorelli \\ cristina.tassorelli@ \\ mondino.it
}

\section{RELATED ARTICLE}

\section{Comment}

Noninvasive

neurostimulation for migraine should be part of the general neurologist's therapeutic armamentarium

Page 167

\section{MORE ONLINE}

$\rightarrow$ Class of Evidence

Criteria for rating

therapeutic and diagnostic

studies

NPub.org/coe

\section{ค Podcast}

Dr. Teshamae Monteith interviews Dr. Cristina

Tassorelli about vagus nerve stimulation for treating migraine.

NPub.org/obi0b6

- CME Course

NPub.org/cmelist

From the Headache Science Centre (C.T.), IRCCS C. Mondino Foundation, Pavia; University of Pavia (C.T.); Headache Center (L.G.), Carlo Besta Neurological Institute and Foundation, Milan; Neurophysiology and Pain Unit (M.d.T.), University of Bari Aldo Moro; Istituto di Ricovero e Cura a Carattere Scientifico (IRCCS) (G.P.), Istituto delle Scienze Neurologiche di Bologna; Department of Clinical and Molecular Medicine (P.M.), Sapienza University, Rome; Department of Neuroscience (I.R.), University of Turin, Italy; MedLogix Communications, LLC (S.D.), Itasca IL; Headache Centre (P.G.), University Hospital of Careggi, Florence; IRCCS Neuromed (A.A.), Pozzilli (IS); Neurologic Clinic (P.S.), Santa Maria della Misericordia Hospital, Perugia, Italy; electroCore, LLC (E.L.), Basking Ridge, NJ; and Headache and Pain Unit (P.B.), IRCCS San Raffaele Pisana, Rome, Italy.

Go to Neurology.org/N for full disclosures. Funding information and disclosures deemed relevant by the authors, if any, are provided at the end of the article. 


\section{Glossary}

$\mathbf{A E}=$ adverse event; $\mathbf{C I}=$ confidence interval; ICHD-3 beta $=$ International Classification of Headache Disorders, 3rd edition (beta version); IHS = International Headache Society; ITT = intent-to-treat; $\mathbf{n V N S}=$ noninvasive vagus nerve stimulation; PRESTO = Prospective Study of nVNS for the Acute Treatment of Migraine; SAE = serious adverse event.

The multiple pharmacologic classes that are effective for the acute treatment of migraine are sometimes limited by lack of availability or risks of drug interactions, medication overuse, and adverse events (AEs). ${ }^{1,2}$ Results from the American Migraine Prevalence and Prevention study revealed no further therapeutic gains of adding or switching to different triptans or other existing treatment classes, ${ }^{1,3}$ and some patients may be dissatisfied with the inconsistency of triptans in adequately treating their attacks. ${ }^{4,5}$ Practical alternatives, such as noninvasive neuromodulation therapies that are effective, safe, and well-tolerated, are attractive for early, adjunct, or frequent use when standard pharmacologic interventions are largely unavailable or ineffective. ${ }^{1,5-7}$

Noninvasive vagus nerve stimulation (nVNS; gammaCore; electroCore, LLC, Basking Ridge, NJ) has shown efficacy in pilot studies of acute migraine treatment and is highly practical and convenient due to its strong safety and tolerability profile. $^{8-11}$ Results from a small open-label study demonstrated 2-hour responder rates of $21 \%$ for pain freedom and $47 \%$ for pain relief after the first treated attack. ${ }^{9}$ Findings indicated that a large randomized controlled study of nVNS as an acute migraine treatment was warranted. Conducting this trial according to International Headache Society (IHS) guidelines was critical in ensuring appropriate scientific rigor and consistency with other comparable studies. ${ }^{12}$ In alignment with these guidelines, objectives of the current study were to (1) compare clinically meaningful outcomes of acute $\mathrm{nVNS}$ treatment with those of a sham device in participants with episodic migraine and (2) evaluate the safety and tolerability of nVNS.

\section{Methods}

\section{Primary research question}

Is nVNS effective for the acute treatment of migraine attacks in episodic migraine? This study provides Class I evidence that for patients with an episodic migraine, nVNS significantly increases the probability of having mild pain or being pain-free 2 hours poststimulation (absolute difference 13.2\%).

\section{Study design}

This multicenter, randomized, double-blind, parallel-group, sham-controlled study took place across 10 Italian sites from January 11, 2016, through March 31, 2017. The study was designed to compare nVNS to sham treatment and included three 4-week periods: (1) a run-in period; (2) a double-blind period; and (3) an open-label period. In the observational run-in period, participants treated their migraine attacks with standard medications according to their individual prescriptions. Participants treated up to 5 migraine attacks with nVNS or sham in the double-blind period and up to 5 additional attacks with nVNS in the open-label period; only 1 attack could be treated in a 48-hour period.

\section{Standard protocol approvals, registrations, and participant consents}

Investigators obtained local ethics committee approval of the protocol, which was registered at ClinicalTrials.gov (NCT02686034). All participants provided written informed consent before study initiation.

\section{Participants}

Study sites enrolled participants who were $18-75$ years of age with a previous diagnosis of migraine with or without aura according to ICHD-3 beta criteria. ${ }^{13}$ Participants were $<50$ years of age at migraine onset and had an attack frequency of 3-8 attacks per month with $<15$ headache days per month over the last 6 months.

Key exclusion criteria were as follows: history of secondary headache, aneurysm, intracranial hemorrhage, brain tumors, significant head trauma, substance abuse, addiction, syncope, or seizure; another significant pain disorder; cardiovascular/ cerebrovascular disease; uncontrolled hypertension; psychiatric/ cognitive disorders; pregnancy; medical condition requiring oral/ injectable steroids; botulinum toxin injections in the past 6 months; head or neck nerve blocks in the past 2 months; previous migraine prevention surgery, cervical vagotomy, electrical device, or metal cervical spine hardware implantation; current use of opioids for more than 2 days per month; current use of simple analgesics or nonsteroidal anti-inflammatory drugs for more than 15 days per month; current use of triptans, ergots, or combined analgesics for more than 10 days per month; and initiation of preventive migraine medications in the past 2 months. Participants who entered the double-blind period were reassessed for entry criteria.

Participants receiving preventive migraine medications at baseline (or other preventive medications determined to potentially interfere with the study) were required to have maintained a stable dose and frequency of these medications during the 2 months before enrollment and throughout the study; initiation of new preventive medications was not permitted during this period.

\section{Randomization and blinding}

Participants were randomly assigned (1:1) to receive $\mathrm{nVNS}$ or sham (variable block design [4 and 6], stratified by site) 
according to independent statistician-generated randomization schedules. A third-party distributor allocated the devices to the sites. Devices were labeled with a serial number and not outwardly identified as active or sham. The Merge eClinical OS Interactive Web Response system provided study site personnel (investigator or designee) with a sequential participant randomization number and corresponding device serial number. A sponsor designee provided the randomization schedule to a site-identified unblinded trainer. The trainer was unblinded to provide participants with instructions that were specific to the assigned device and had no further interaction with participants. This allowed for investigators and participants to remain blinded to treatment assignments throughout the study in order to minimize potential bias related to the device-specific training instructions.

\section{Interventions}

The nVNS device produces a proprietary low-voltage electrical signal comprising a $5-\mathrm{kHz}$ sine wave burst lasting for 1 ms ( 5 sine waves, each lasting $200 \mu \mathrm{s}$ ), with such bursts repeated once every $40 \mathrm{~ms}(25 \mathrm{~Hz})$, generating a $24-\mathrm{V}$ peak voltage and $60-\mathrm{mA}$ peak output current. The sham device produces a low-frequency $(0.1 \mathrm{~Hz})$ biphasic signal that was intended to be physically perceived without actually stimulating the vagus nerve or generally causing muscle contraction.

An unblinded trainer instructed the participants on electronic diary completion and correct study device use. A follow-up phone call at 5 days into each study period ensured adherence to the instructions. Within 20 minutes from migraine pain onset, participants self-administered bilateral 120-second stimulations to the right and left sides of the neck. Participants recorded posttreatment assessments in their diaries at 15, 30, 60, and 120 minutes and 24 and 48 hours after completion of the initial bilateral stimulations. Participants were instructed to repeat the bilateral stimulations if pain had not improved at the 15-minute assessment, and participants who were not pain-free at the 120minute assessment had the option of administering an additional set of bilateral stimulations. Participants were asked to wait 120 minutes before using acute rescue medication.

\section{Study endpoints}

As recommended by IHS guidelines, ${ }^{14}$ the primary efficacy endpoint was the proportion of participants who were painfree without using rescue medication at 120 minutes after study treatment completion for the first treated migraine attack of the double-blind period. Key secondary and other efficacy endpoints for the first treated attack during the double-blind period included pain-free rates at 30 and 60 minutes; pain relief at 30, 60, and 120 minutes; mean percentage change in pain score from baseline to 30,60 , and 120 minutes; and the absence of associated symptoms (i.e., nausea, vomiting, photophobia, and phonophobia) at 120 minutes. Pain relief was defined according to the IHS guidelines for controlled studies of migraine medications as a decrease in pain intensity from moderate (2) or severe (3) to mild (1) or no (0) pain on a 4-point scale. ${ }^{14}$ Consistency of response, another clinically meaningful outcome recommended

Figure 1 Participant disposition

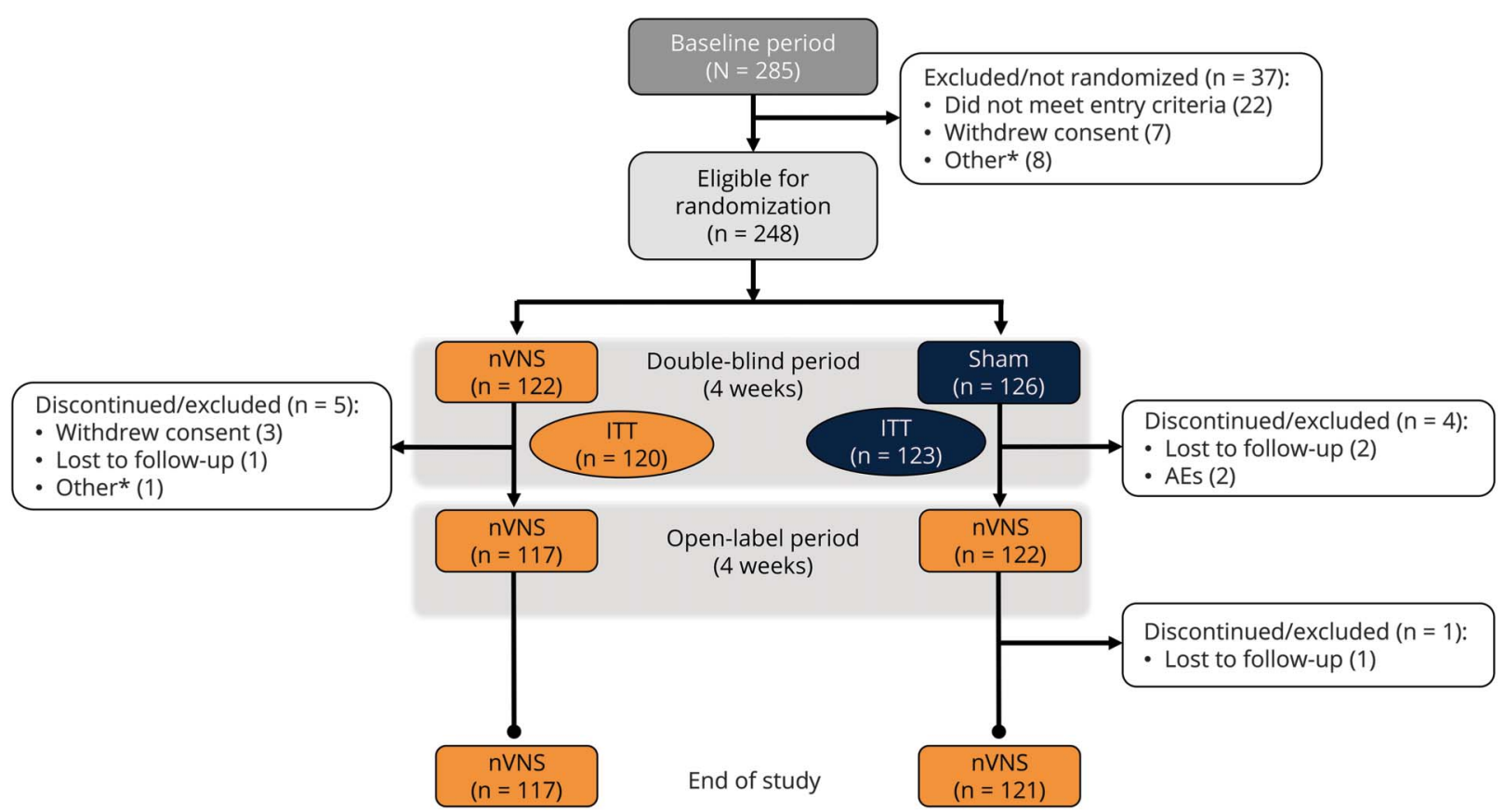

$\mathrm{AE}$ = adverse event; ITT = intent-to-treat; nVNS = noninvasive vagus nerve stimulation. *Other reasons for discontinuation included inability to fulfill visits because of injury, inability to continue the study because of family commitments, dissatisfaction with or discontinued/lack of use of the device, and noncompliance with study procedures. 
by IHS guidelines, ${ }^{14}$ was evaluated during the double-blind period by calculating $\geq 50 \%$ responder rates at 120 minutes for both pain freedom and relief in those with at least 2 treated migraine attacks. Pain freedom and pain relief were also evaluated at 120 minutes after the first treated attack in the open-label period, as was the consistency of response for those who treated at least 2 attacks during this period. Additional outcomes included blinding effectiveness, participant satisfaction (1, extremely satisfied; 5 , not at all satisfied), participant willingness to recommend the device to a friend or family member, and ease of device use ( 1 , very easy; 4 , very difficult). The safety and tolerability of nVNS was assessed by comparing rates of AEs, adverse device effects, and serious adverse events (SAEs) among the nVNS group and controls.

\section{Statistical methods}

A sample size of 232 participants ( 116 per treatment arm) was determined to provide $90 \%$ power to demonstrate statistical significance for the primary endpoint, assuming a sham painfree rate of $18 \%$, a treatment difference of $20 \%$, an a value of 0.05 , and an attrition rate of $10 \%$.

Descriptive statistics were used for both continuous variables (means and 95\% confidence intervals [CIs]) and categorical variables (frequency counts, percentages, and 95\% CIs). Each attack was assessed separately, with responder rates based solely on pain intensity if medication use data were missing. Missing pain intensity data were imputed using the last observation carried forward. Participants who did not provide data on associated symptoms were excluded from symptomspecific analyses.

Statistical efficacy analyses (nVNS vs control) were conducted on the intent-to-treat (ITT) population, defined as all randomized participants who treated at least 1 migraine attack in the double-blind period. A logistic regression analysis was

Table 1 Demographics and participant/attack characteristics

\begin{tabular}{|c|c|c|}
\hline Characteristic & $n V N S(n=120)$ & Sham $(n=123$ \\
\hline \multicolumn{3}{|l|}{ At baseline } \\
\hline Age, y & $38.8 \pm 11.0$ & $39.6 \pm 11.8$ \\
\hline Age at migraine onset, $y$ & $29.4 \pm 11.2$ & $28.5 \pm 11.5$ \\
\hline Female, n (\%) & $95(79.2)$ & $91(74.0)$ \\
\hline Caucasian, $\mathbf{n}(\%)$ & $120(100.0)$ & $123(100.0)$ \\
\hline \multicolumn{3}{|l|}{ Migraine type, $n(\%)$} \\
\hline Migraine with aura & $8(6.7)$ & $9(7.3)$ \\
\hline Migraine without aura & $112(93.3)$ & $114(92.7)$ \\
\hline Attacks in the last 4 weeks, $n$ & $5.4 \pm 1.7$ & $5.3 \pm 1.7$ \\
\hline Headache days in the last 4 weeks, $n$ & $6.3 \pm 2.3$ & $6.2 \pm 2.1$ \\
\hline Attacks per month in the last 6 months, $n$ & $5.4 \pm 1.5$ & $5.4 \pm 1.5$ \\
\hline Acute migraine medication use per month, $d$ & $5.6 \pm 1.7$ & $5.3 \pm 1.7$ \\
\hline Preventive medication use, $\mathrm{n}(\%)$ & $42(35.0)$ & $35(28.5)$ \\
\hline \multicolumn{3}{|l|}{ At attack onset $^{\mathrm{a}}$} \\
\hline \multicolumn{3}{|c|}{ Migraine attack severity (first treated attack), n (\%) } \\
\hline Mild & $40(33.6)$ & $46(38.7)$ \\
\hline Moderate & $51(42.9)$ & $55(46.2)$ \\
\hline Severe & $28(23.5)$ & $18(15.1)$ \\
\hline \multicolumn{3}{|c|}{ Migraine attack severity (all treated attacks), $\mathrm{n}(\%)^{\mathbf{b}}$} \\
\hline Mild & $113(31.5)$ & $105(31.9)$ \\
\hline Moderate & $156(43.5)$ & $166(50.5)$ \\
\hline Severe & $90(25.1)$ & $58(17.6)$ \\
\hline
\end{tabular}

Abbreviation: nVNS = noninvasive vagus nerve stimulation.

a Participants with no reported severity at attack onset are excluded from this analysis.

${ }^{b}$ First treated attack: nVNS, $n=119$; sham, $n=119 ;$ all treated attacks: $n V N S, n=359$; sham, $n=329$. 
included in the primary endpoint (pain freedom at 120 minutes for the first attack in the double-blind period) with adjustment for the participants' baseline pain score, use of preventive therapies, and presence of aura and was repeated for the 30 - and 60-minute time points; the presence of aura was not considered in the 30-minute analysis because of model fit issues. A post hoc repeated-measures analysis using generalized linear mixedeffects regression models was conducted to gain further insight from all of the data collected at multiple time points through the 120-minute time point of the primary efficacy analysis. The repeated-measures analysis was also adjusted for baseline pain score, use of preventive therapies, and presence of aura. Mean percentage changes in pain score were compared between treatment groups using 2 -sample $t$ tests. The remaining secondary and other analyses reported were evaluated using the $\chi^{2}$ test or Fisher exact test, as appropriate. Two-sided $p$ values $<0.05$ were considered statistically significant. Use of rescue medication before the 120-minute assessment was considered a study treatment failure.

Blinding analyses were performed using the Bang blinding index. Safety analyses were conducted on the safety population, defined as all enrolled participants. All data were analyzed using SAS 9.4 (SAS Institute Inc., Cary, NC).

\section{Data availability}

Any data not published within this article will be publicly available at ClinicalTrials.gov with the identifier NCT02686034. Individual participant data will not be shared.

\section{Results}

\section{Participants}

In the Prospective Study of nVNS for the Acute Treatment of Migraine (PRESTO), 285 participants were enrolled, 248 were randomized, and 243 formed the ITT population (figure 1). A total of 237 participants from the ITT population completed the open-label period, with 219 of these treating at least 1 attack during this period. Demographic and baseline characteristics were generally well-balanced between the nVNS group and controls (table 1). A higher proportion of participants in the nVNS group treated their first attack when its intensity was severe $(p=0.254)$ and were using preventive medications $(p=0.273)$. The most commonly used

Figure 2 Responder rates after the first treated attack during the double-blind period
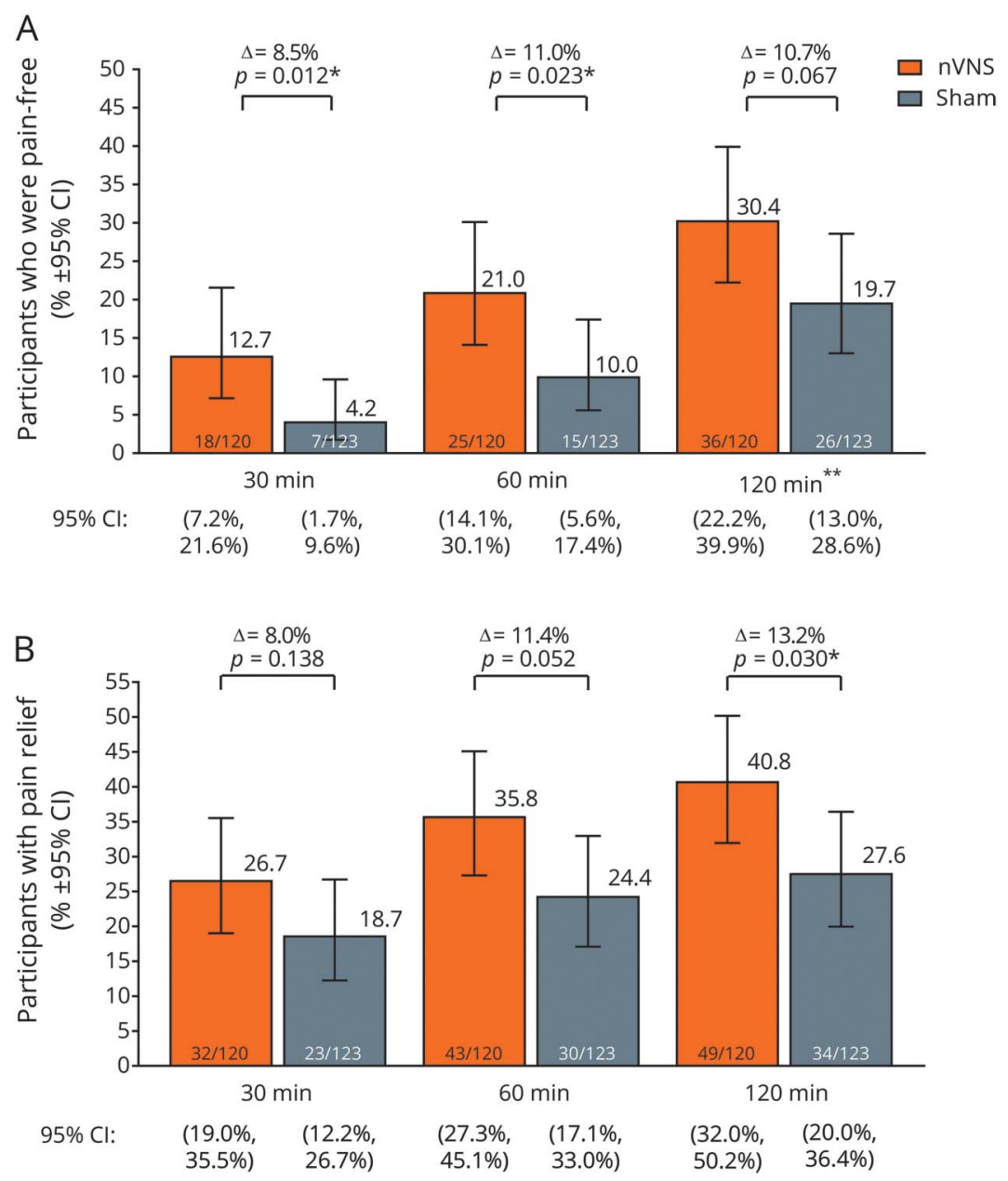

(A) Pain freedom and (B) pain relief. $\mathrm{Cl}=$ confidence interval; nVNS = noninvasive vagus nerve stimulation Data for no. of participants in (A) are unadjusted numbers. *Statistically significant. **Primary endpoint. 
preventive medications were topiramate, vitamin $B_{2}$, magnesium, and propranolol.

\section{Pain-free responder rates}

For the first treated migraine attack, the proportion of participants who became pain-free was significantly higher with nVNS than with sham at 30 and 60 minutes but not at 120 minutes (primary endpoint) (figure 2A). A repeatedmeasures test examined the inconsistency between the 120minute finding and the 30- and 60-minute findings and found nVNS to be superior to sham for the pain-free outcome through 30, 60, and 120 minutes (odds ratio 2.3; 95\% CI 1.2, 4.4; $p=0.012$ ).

\section{Responder rates for pain relief}

Responder rates for pain relief at 120 minutes were significantly higher with nVNS than with sham for the first treated migraine attack (figure 2B). Responder rates for pain relief were numerically higher with nVNS at 30 and 60 minutes, but these differences were not significant (figure 2B).

\section{Percentage pain intensity reductions}

Mean percentage pain intensity reductions for the first attack were significantly more pronounced with nVNS than with sham at 60 and 120 minutes (figure 3 ).

\section{Consistency of response}

Proportions of participants who responded at 120 minutes for $\geq 50 \%$ of their attacks were significantly higher with nVNS than with sham for both pain freedom and pain relief (figure 4).

\section{Associated symptoms}

As expected with the early treatment of attacks in this study, relatively low percentages of participants were experiencing

Figure 3 Mean percentage pain score reductions

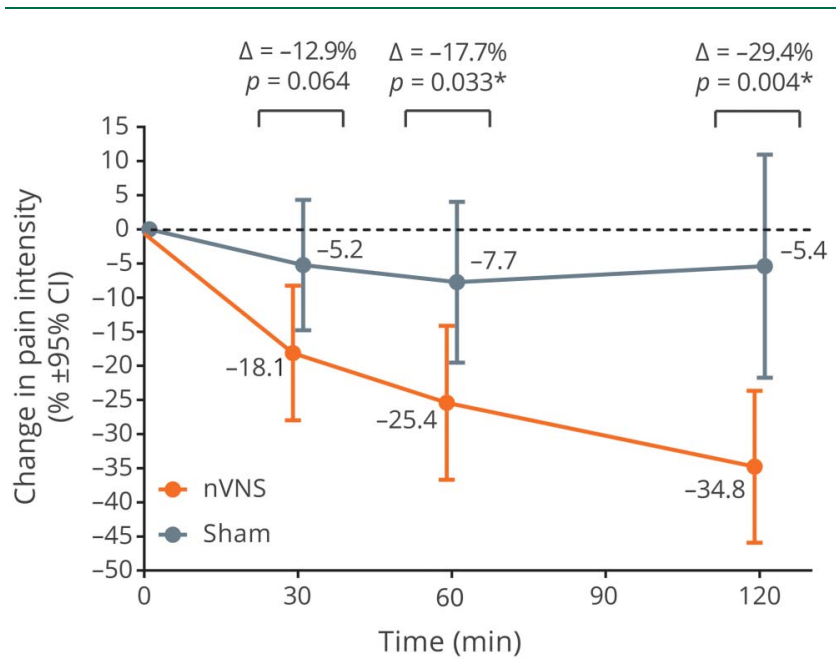

Changes are from baseline for the first treated attack in the double-blind period; post hoc analysis. $\mathrm{Cl}$ = confidence interval; nVNS = noninvasive vagus nerve stimulation. *Statistically significant. an associated symptom at the time of initial treatment (vomiting, 6.6\%; nausea, 30.5\%; photophobia, 46.9\%; phonophobia, $43.2 \%)$. The number of participants who did not report vomiting at 120 minutes after the first treated attack was higher in the nVNS group $(6 / 8$ [75.0\%]) than in controls $(3 / 8[37.5 \%])(p=0.315)$. Proportions of participants who became nausea-free (nVNS, 14/35 [40.0\%]; sham, $16 / 39$ [41.0\%]; $p=0.929)$, photophobia-free (nVNS, 20/53 [37.7\%]; sham, 23/61 [37.7\%]; $p=$ 0.997), or phonophobia-free (nVNS, 16/48 [33.3\%]; sham, 24/57 [42.1\%]; $p=0.357)$ at 120 minutes after the first treated attack were similar among the $n V N S$ group and controls.

\section{Blinding}

After the first treatment and at the end of the double-blind period, participants indicated the treatment they thought they had received (nVNS, sham, do not know); guesses were generally similar among the nVNS group and controls (figure 5). After the first treatment, Bang blinding index estimates for $\mathrm{nVNS}$ and sham indicated successful blinding (i.e., inclusion of zero in the $95 \% \mathrm{CI}$ ) (figure 5).

\section{Device use and perceptions}

Almost all participants (98\%) administered at least 1 stimulation and were adherent to the treatment instructions, but most participants did not administer repeat stimulations for the first attack at 15 minutes as instructed (nVNS, 60.8\%; sham, $60.2 \%$ ) or optionally at 120 minutes (nVNS, 95.8\%; sham, $93.5 \%)$.

The percentage of participants who rated the treatment as a little satisfying or better at the end of the double-blind period was higher for nVNS (72.0\%) than for sham (63.9\%), as was the percentage of participants who would recommend their study device to a friend or family member (nVNS, $60.2 \%$; sham, $46.7 \%$ ). Approximately $96 \%$ of participants in each treatment group reported that their device was somewhat or very easy to use.

\section{Efficacy findings from the open-label period}

Therapeutic benefits seen with nVNS in the double-blind period were sustained after an additional 4 weeks of acute nVNS use in the open-label period. Findings were consistent after the first treated attack of the double-blind and open-label periods for pain-free response $(30.4 \%$ and $27.9 \%$, respectively) and pain relief (40.8\% and $43.4 \%$, respectively). The consistency of response to nVNS was also maintained from the double-blind period (pain freedom, 32.4\%; pain relief, $47.6 \%$ ) to the open-label period (pain freedom, 26.8\%; pain relief, $41.8 \%$ ).

\section{Safety and tolerability}

Acute nVNS therapy demonstrated a highly favorable safety and tolerability profile (table 2 ). The most common AEs were application site discomfort and nasopharyngitis 
Figure $4 \geq 50 \%$ responder rates at 120 minutes during the double-blind period

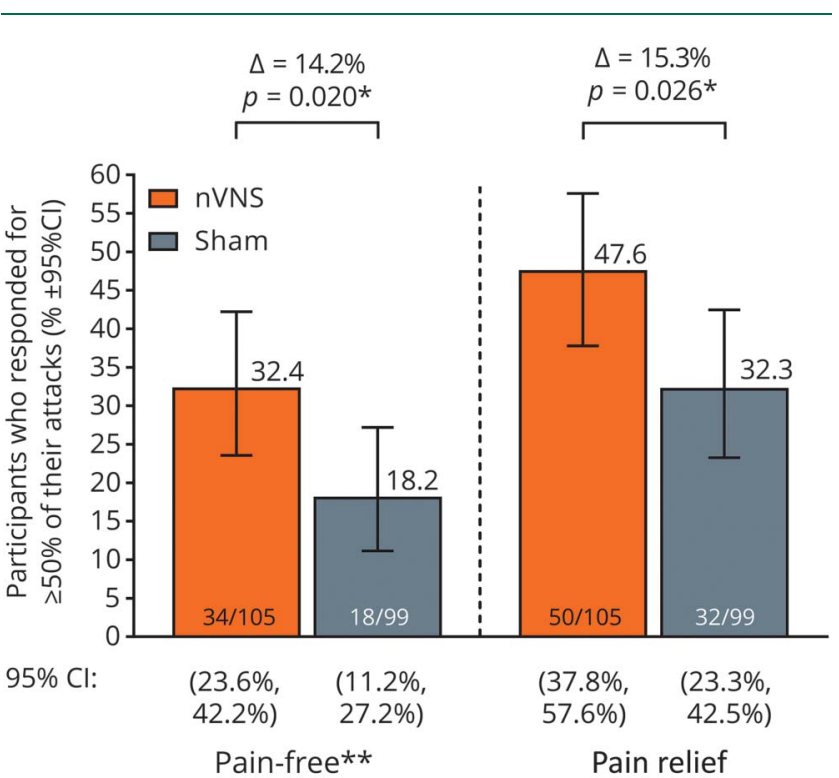

$\mathrm{Cl}=$ confidence interval; nVNS = noninvasive vagus nerve stimulation. *Statistically significant. **Post hoc analysis.

in the nVNS group and application site erythema and pain, dizziness, flu-like symptoms, and nasopharyngitis among controls. Participants reported no SAEs during the study. Only 2 participants, both controls, discontinued from the study due to AEs.

\section{Discussion}

The PRESTO trial is one of the largest randomized, doubleblind, sham-controlled studies of a noninvasive neuromodulation device for the acute treatment of migraine. ${ }^{2}$ In the absence of guidelines for evaluating neuromodulation in migraine, this trial was designed and conducted using stringent IHS recommendations for evaluating migraine medications. ${ }^{14}$ Our adherence to the current standards for pharmacologic trials included the use of 2-hour pain freedom as the primary endpoint, which is less vulnerable to the placebo/sham effect compared with pain relief. ${ }^{44,15}$

nVNS was superior to sham in aborting the first treated attack at 30 and 60 minutes but not at 120 minutes. A repeated-measures test provided additional insight into the primary endpoint by supporting the superiority of $\mathrm{nVNS}$ over sham in achieving pain freedom through 30,60, and 120 minutes. The pain-free responder rate at 120 minutes in the nVNS group (30.4\%) was consistent with those generally seen in previous studies evaluating the efficacy of oral triptans $(14 \%-42 \%)^{4,16-18}$ and of potent nonsteroidal anti-inflammatory drugs $(15 \%-25 \%){ }^{19-21}$ Higher 2-hour pain-free rates of oral triptans (47\%-68\%) have been reported in patients with mild-intensity migraines who also generally had higher 2-hour pain-free placebo rates and in patients treated with higher-dose regimens, ${ }^{17}$ which often further increase the risk of adverse effects. ${ }^{22}$ Taken together, findings of this large, rigorous, well-controlled trial strongly suggest that $\mathrm{nVNS}$ is effective and has benefits consistent with standard drug options but with the added benefit of an extremely benign adverse effect profile and the flexibility to be used for multiple attacks without the risk of medication overuse or drug-related adverse effects. As a nonpharmacologic option, nVNS can also be utilized in combination with any existing medication.

A higher percentage of participants in the nVNS group treated their first attack while it was severe in intensity. This difference, although not statistically significant, suggests a clinically meaningful bias toward more severe pain at the time of treatment in the nVNS group that may have moderated the therapeutic gain seen in the study results. Associated symptoms had not yet emerged in many participants as nVNS or sham treatment was initiated within 20 minutes from migraine pain onset, which limits statistical power for evaluating these symptoms. The early treatment design likely contributed to the lack of superiority of nVNS in improving associated symptoms, consistent with challenges in other noninvasive device studies. ${ }^{23,24}$ Treatment responses may have also been mitigated due to the limited use of repeat stimulations. Additional stimulations appeared to improve efficacy in previous studies and carry minimal risks given the favorable safety and tolerability of nVNS. ${ }^{11,25-29}$ A possible explanation for why most participants in the current study did not administer repeat stimulations as instructed may involve a perceived rapid pain relief from the initial stimulations that led to a lack of need or motivation to administer the additional stimulations.

The limitations of this study include the selection of an appropriate sham device, which is a consistent challenge in neuromodulation studies. ${ }^{30}$ The sham device in this study had an active signal that was strong enough to be perceived but was not intended to stimulate the vagus nerve, as recommended in published literature. ${ }^{30}$ The apparent strength of the sham signal helped to maintain blinding but could have conceivably elevated the effects of sham treatment across all endpoints, limiting the ability of clinically meaningful active therapeutic gains to achieve statistical superiority. We hypothesize that the higher-than-expected sham results could represent either a psychobiological placebo effect ${ }^{31}$ or a physiologically active response potentially related to an unanticipated low level of vagal or other activity generated by an active sham signal being applied to the neck.

The mechanism of action of $\mathrm{nVNS}$ in the treatment of migraine is largely elusive and likely multifactorial. Preclinical data have demonstrated that this therapy inhibits the trigeminovascular pathway and suppresses extracellular glutamate in the central nervous system. ${ }^{25,32}$ A study that used a validated migraine-specific animal model demonstrated that direct activation of the vagus nerve, which has been shown to be 
Figure 5 Blinding

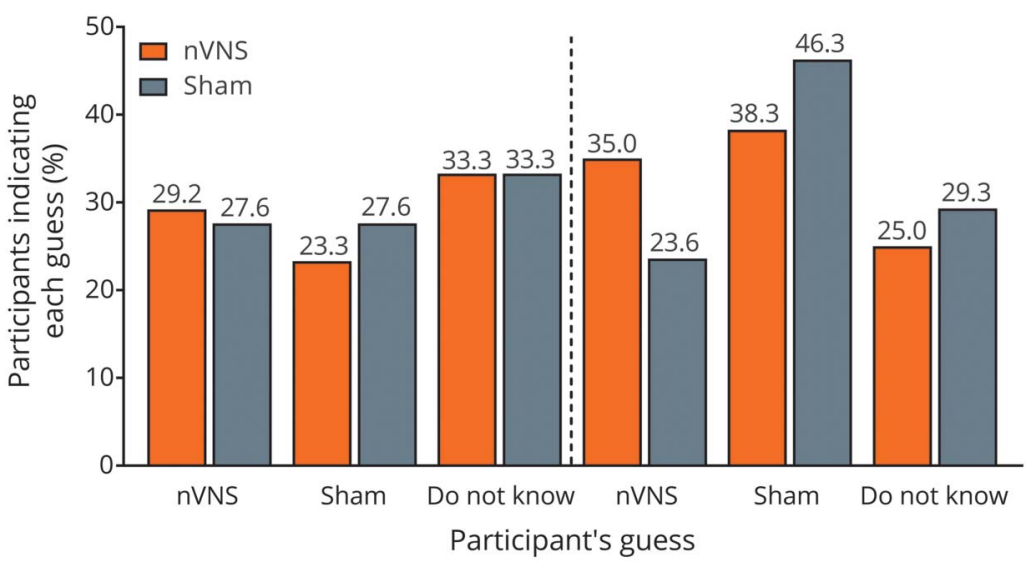

120 min after first attack Bang blinding index $(95 \% \mathrm{Cl})$ : nVNS: $0.07(-0.04,0.17)$ * Sham: $0(-0.11,0.11)^{*}$
End of double-blind period Bang blinding index $(95 \% \mathrm{Cl})$ : nVNS: $-0.03(-0.14,0.08)$ *

Sham: $0.23(0.13,0.33)$
nVNS = noninvasive vagus nerve stimulation. *Inclusion of zero in the $95 \%$ confidence interval $(\mathrm{Cl})$ indicates successful blinding. elicited by nVNS, ${ }^{33,34}$ suppressed the acute nociceptive activation of trigeminocervical neurons. ${ }^{25}$ In a separate study using an established rodent model of chronic trigeminal allodynia, nVNS reversed the elevation in extracellular glutamate, a marker of increased pain, within the trigeminal nucleus caudalis. ${ }^{32}$ These studies suggest that direct activation of the vagus nerve by nVNS treatment facilitates central inhibition in the trigeminovascular system, contributing to the clinical efficacy seen in the current study.
The scientific rigor of the large PRESTO trial and its adherence to IHS guidelines for pharmacologic studies allow for the consideration of nVNS in the context of currently available acute treatment options for migraine. ${ }^{12,14,17}$ Our results provide an option for both headache centers and the larger migraine community to address the need for further optimization of the control of migraine attacks without any added adverse effects or the risk of medication overuse. ${ }^{35}$ PRESTO and a forthcoming randomized sham-controlled

Table 2 Incidence of adverse events (AEs) and adverse device effects (ADEs) (all study periods)

\begin{tabular}{lll}
\hline AEs and ADEs & nVNS $(\mathbf{n = 1 2 2 )}$ & Sham (n=126) \\
\hline$\geq 1$ AE & $22(18.0)$ & $23(18.3)$ \\
\hline$\geq 1$ ADE & $7(5.7)$ & $10(7.9)$ \\
\hline$\geq 1$ SAE & 0 & \\
\hline AEs occurring in $\geq 2 \%$ of participants in any treatment group & & \\
\hline General disorders and administration site conditions & $3(2.5)$ & $3(0.8)$ \\
\hline Application site discomfort & 0 & $3(2.4)$ \\
\hline Application site erythema & 0 & $3(2.4)$ \\
\hline Application site pain & & $3(2.4)$ \\
\hline Infections and infestations & 0 & $3(2.4)$ \\
\hline Influenza & $2(1.6)$ & $3(2.4)$ \\
\hline Nasopharyngitis & & \\
\hline Nervous system disorders & 0 & \\
\hline Dizziness
\end{tabular}

Abbreviations: $\mathrm{nVNS}$ = noninvasive vagus nerve stimulation; $\mathrm{SAE}=$ serious adverse event.

Safety population. Data are $\mathrm{n}(\%)$ of participants. 
study of nVNS for the prevention of episodic migraine (ClinicalTrials.gov identifier: NCT02378844) may also help the migraine community by informing future recommendations regarding neuromodulation trials.

nVNS is a novel, flexible therapeutic approach for the acute treatment of migraine attacks. The therapeutic benefit of nVNS was consistent across several important, clinically relevant endpoints and was similar to that of oral triptans, as demonstrated by similar 2-hour pain-free responder rates. ${ }^{4,16,18}$ Its combination of efficacy and consistent safety and tolerability, established over multiple studies, ${ }^{11,26-29}$ makes it an attractive option for early and frequent use. nVNS may be used in conjunction with existing treatments without any drug interactions or as monotherapy, potentially delaying or decreasing the need for medications that can be limited by lack of availability, inconvenience, or AEs. ${ }^{1,2,5,36}$ nVNS offers a convenient complementary treatment option that is easy to use, has no pharmacologic AEs, and preserves the flexibility to use standard medications when needed. Findings from this randomized sham-controlled trial demonstrate the efficacy of nVNS for aborting attacks as early as 30 minutes and up to 60 minutes and for relieving pain at 120 minutes in the acute treatment of episodic migraine with or without aura. This study also confirms the practicality and strong safety and tolerability profile of nVNS.

\section{Author contributions}

Cristina Tassorelli, Licia Grazzi, and Eric Liebler contributed to the PRESTO study design and provided detailed input into the development of the manuscript. All primary investigators were involved in participant recruitment and treatment for the PRESTO study. All authors participated in data collection, interpretation, and validation and had full access to all study data. Cristina Tassorelli and Eric Liebler were involved in data analysis. Data analysis support from North American Science Associates was funded by electroCore, LLC. Cristina Tassorelli, Eric Liebler, and Stefanie Dorlas drafted and revised the manuscript for content in cooperation with all authors. All authors reviewed, critiqued, and contributed to revision of the manuscript content and provided approval of the final manuscript draft to be submitted to Neurology ${ }^{\circledR}$. Professional medical writing and editorial support (i.e., technical editing, copyediting, preparation of tables and figures, and clerical assistance) from MedLogix Communications, LLC, was funded by electroCore, LLC. The principal author, Cristina Tassorelli, takes responsibility for the data, analyses and interpretation, and conduct of the research.

\section{Acknowledgment}

The authors acknowledge all coinvestigators, research nurses, study sites, and electroCore study team members. Statistical analyses for the study were conducted by Candace McClure, $\mathrm{PhD}$, and Lisa Thackeray, MS, of North American Science Associates Inc. (Minneapolis, MN). Medical writing support was provided by Stefanie Dorlas, BMath, BEd, of MedLogix Communications, LLC, in cooperation with the authors.

\section{Study funding}

This study was sponsored by electroCore, LLC.

\section{Disclosure}

C. Tassorelli has received consultancy fees from Allergan S.p.A., electroCore, LLC, Eli Lilly and Company, and Novartis AG and research grants from the European Commission and the Italian Ministry of Health. She is also a principal investigator or collaborator for RCTs sponsored by Alder BioPharmaceuticals Inc., Eli Lilly and Company, and Teva Pharmaceutical Industries Ltd. L. Grazzi has received consultancy and advisory fees from Allergan S.p.A. and electroCore, LLC. M. de Tommaso has received advisory fees from Allergan S.p.A., Neopharmed, and Pfizer Inc. G. Pierangeli reports no disclosures relevant to the manuscript. P. Martelletti has received research grants, advisory board fees, or travel fees from ACRAF, Allergan S.p.A., Amgen Inc., electroCore, LLC, Novartis AG, and Teva Pharmaceutical Industries Ltd. I. Rainero has received consultancy fees from electroCore, LLC, and Mylan N.V. and research grants from the European Commission-Horizon 2020. He is also a principal investigator for RCTs sponsored by Axovant Sciences Ltd. and TauRx Pharmaceuticals Ltd. S. Dorlas is an employee of MedLogix Communications, LLC, as a senior medical writer. P. Geppetti has received consultancy fees from Allergan S.p.A., electroCore, LLC, Evidera, Novartis AG, Pfizer Inc., and Sanofi S.p.A. and research grants from Chiesi Farmaceutici S.p.A. He is also a principal investigator for RCTs sponsored by Eli Lilly and Company, Novartis AG, and Teva Pharmaceutical Industries Ltd. A. Ambrosini has received consultancy fees from Almirall, S.A., and travel grants from Allergan S.p.A. and Almirall S.A. P. Sarchielli has received clinical study fees from Allergan S.p.A. E. Liebler is an employee of electroCore, LLC, and receives stock ownership. P. Barbanti has received consultancy fees from Allergan S.p.A., electroCore, LLC, Janssen Pharmaceuticals, Inc., Lusofarmaco, and Visufarma and advisory fees from Abbott Laboratories and Merck \& Co., Inc. Go to Neurology.org/ $\mathrm{N}$ for full disclosures.

Received January 2, 2018. Accepted in final form April 11, 2018.

\section{References}

1. Buse DC, Serrano D, Reed ML, et al. Adding additional acute medications to a triptan regimen for migraine and observed changes in headache-related disability: results from the American Migraine Prevalence and Prevention (AMPP) study. Headache 2015;55:825-839.

2. Puledda F, Goadsby PJ. An update on non-pharmacological neuromodulation for the acute and preventive treatment of migraine. Headache 2017;57:685-691.

3. Serrano D, Buse DC, Kori SH, et al. Effects of switching acute treatment on disability in migraine patients using triptans. Headache 2013;53:1415-1429.

4. Ferrari MD, Goadsby PJ, Roon KI, Lipton RB. Triptans (serotonin, 5-HT1B/1D agonists) in migraine: detailed results and methods of a meta-analysis of 53 trials. Cephalalgia 2002;22:633-658.

5. Lipton RB, Buse DC, Serrano D, Holland S, Reed ML. Examination of unmet treatment needs among persons with episodic migraine: results of the American Migraine Prevalence and Prevention (AMPP) study. Headache 2013;53:1300-1311.

6. Bigal ME, Serrano D, Buse D, Scher A, Stewart WF, Lipton RB. Acute migraine medications and evolution from episodic to chronic migraine: a longitudinal population-based study. Headache 2008;48:1157-1168.

7. Yuan H, Silberstein SD. Vagus nerve stimulation and headache. Headache 2017;57: 29-33.

8. Barbanti P, Grazzi L, Egeo G, Padovan AM, Liebler E, Bussone G. Non-invasive vagus nerve stimulation for acute treatment of high-frequency and chronic migraine: an open-label study. J Headache Pain 2015;16:61. 
9. Goadsby PJ, Grosberg BM, Mauskop A, Cady R, Simmons KA. Effect of noninvasive vagus nerve stimulation on acute migraine: an open-label pilot study. Cephalalgia 2014;34:986-993.

10. Kinfe TM, Pintea B, Muhammad S, et al. Cervical non-invasive vagus nerve stimulation (nVNS) for preventive and acute treatment of episodic and chronic migraine and migraine-associated sleep disturbance: a prospective observational cohort study. J Headache Pain 2015;16:101.

11. Silberstein SD, Calhoun AH, Lipton RB, et al. Chronic migraine headache prevention with noninvasive vagus nerve stimulation: the EVENT study. Neurology 2016;87:529-538.

12. Hougaard A, Tfelt-Hansen P. Are the current IHS guidelines for migraine drug trials being followed? J Headache Pain 2010;11:457-468.

13. The International Classification of Headache Disorders, 3rd edition (beta version). Cephalalgia 2013;33:629-808.

14. Tfelt-Hansen P, Pascual J, Ramadan N, et al. Guidelines for controlled trials of drugs in migraine: third edition: a guide for investigators. Cephalalgia 2012;32:6-38.

15. Voss T, Lipton RB, Dodick DW, et al. A phase IIb randomized, double-blind, placebocontrolled trial of ubrogepant for the acute treatment of migraine. Cephalalgia 2016; 36:887-898.

16. Ferrari MD, Roon KI, Lipton RB, Goadsby PJ. Oral triptans (serotonin 5-HT(1B/ 1D) agonists) in acute migraine treatment: a meta-analysis of 53 trials. Lancet 2001; 358:1668-1675.

17. Marmura MJ, Silberstein SD, Schwedt TJ. The acute treatment of migraine in adults: the American Headache Society evidence assessment of migraine pharmacotherapies. Headache 2015;55:3-20.

18. Silberstein SD, Newman LC, Marmura MJ, Nahas SJ, Farr SJ. Efficacy endpoints in migraine clinical trials: the importance of assessing freedom from pain. Curr Med Res Opin 2013;29:861-867.

19. Brandes JL, Kudrow D, Stark SR, et al. Sumatriptan-naproxen for acute treatment of migraine: a randomized trial. JAMA 2007;297:1443-1454.

20. Diener HC, Montagna P, Gacs G, et al. Efficacy and tolerability of diclofenac potassium sachets in migraine: a randomized, double-blind, cross-over study in comparison with diclofenac potassium tablets and placebo. Cephalalgia 2006;26:537-547.

21. Lipton RB, Grosberg B, Singer RP, et al. Efficacy and tolerability of a new powdered formulation of diclofenac potassium for oral solution for the acute treatment of migraine: results from the International Migraine Pain Assessment Clinical Trial (IMPACT). Cephalalgia 2010;30:1336-1345.

22. Hougaard A, Tfelt-Hansen P. Review of dose-response curves for acute antimigraine drugs: triptans, 5-HT1F agonists and CGRP antagonists. Expert Opin Drug Metab Toxicol 2015;11:1409-1418
23. Lipton RB, Dodick DW, Silberstein SD, et al. Single-pulse transcranial magnetic stimulation for acute treatment of migraine with aura: a randomised, double-blind, parallel-group, sham-controlled trial. Lancet Neurol 2010;9:373-380.

24. Schoenen J, Vandersmissen B, Jeangette S, et al. Migraine prevention with a supraorbital transcutaneous stimulator: a randomized controlled trial. Neurology 2013;80: 697-704.

25. Akerman S, Simon B, Romero-Reyes M. Vagus nerve stimulation suppresses acute noxious activation of trigeminocervical neurons in animal models of primary headache. Neurobiol Dis 2017;102:96-104.

26. Gaul C, Diener HC, Silver N, et al. Non-invasive vagus nerve stimulation for prevention and acute treatment of chronic cluster headache (PREVA): a randomised controlled study. Cephalalgia 2016;36:534-546.

27. Goadsby PJ, de Coo IF, Silver N, et al. Non-invasive vagus nerve stimulation for the acute treatment of episodic and chronic cluster headache: a randomized, doubleblind, sham-controlled ACT2 study. Cephalalgia 2018;38:959-969.

28. Grazzi L, Egeo G, Calhoun AH, McClure CK, Liebler E, Barbanti P. Non-invasive vagus nerve stimulation (nVNS) as mini-prophylaxis for menstrual/menstrually related migraine: an open-label study. J Headache Pain 2016;17:91.

29. Silberstein SD, Mechtler LL, Kudrow DB, et al. Non-invasive vagus nerve stimulation for the acute treatment of cluster headache: findings from the randomized, doubleblind, sham-controlled ACT1 study. Headache 2016;56:1317-1332.

30. Asano E, Goadsby PJ. How do we fashion better trials for neurostimulator studies in migraine? Neurology 2013;80:694.

31. Benedetti F, Carlino E, Pollo A. How placebos change the patient's brain. Neuropsychopharmacology 2011;36:339-354.

32. Oshinsky ML, Murphy AL, Hekierski H Jr, Cooper M, Simon BJ. Noninvasive vagus nerve stimulation as treatment for trigeminal allodynia. Pain 2014;155: 1037-1042.

33. Frangos E, Komisaruk BR. Access to vagal projections via cutaneous electrical stimulation of the neck: fMRI evidence in healthy humans. Brain Stimul 2017;10: 19-27.

34. Nonis R, D'Ostilio K, Schoenen J, Magis D. Evidence of activation of vagal afferents by non-invasive vagus nerve stimulation: an electrophysiological study in healthy volunteers. Cephalalgia 2017;37:1285-1293.

35. Westergaard ML, Steiner TJ, MacGregor EA, et al. The Headache Under-Response to Treatment (HURT) Questionnaire: assessment of utility in headache specialist care. Cephalalgia 2013;33:245-255.

36. Puledda F, Messina R, Goadsby PJ. An update on migraine: current understanding and future directions. J Neurol 2017;264:2031-2039. 


\section{Neurology}

Noninvasive vagus nerve stimulation as acute therapy for migraine: The randomized PRESTO study

Cristina Tassorelli, Licia Grazzi, Marina de Tommaso, et al. Neurology 2018;91;e364-e373 Published Online before print June 15, 2018

DOI 10.1212/WNL.0000000000005857

This information is current as of June 15, 2018

Neurology ${ }^{\circledR}$ is the official journal of the American Academy of Neurology. Published continuously since 1951, it is now a weekly with 48 issues per year. Copyright $@ 2018$ The Author(s). Published by Wolters Kluwer Health, Inc. on behalf of the American Academy of Neurology.. All rights reserved. Print ISSN: 0028-3878. Online ISSN: 1526-632X.

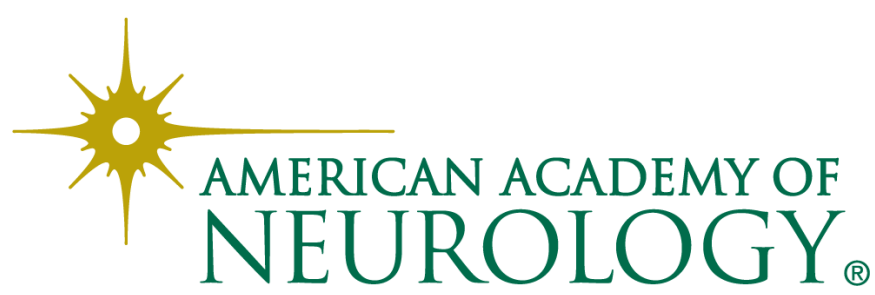




\section{Updated Information \& Services}

\section{Supplementary Material}

References

Citations

Subspecialty Collections

\section{Permissions \& Licensing}

Reprints including high resolution figures, can be found at: http://n.neurology.org/content/91/4/e364.full

Supplementary material can be found at: http://n.neurology.org/content/suppl/2018/07/30/WNL.0000000000005 857.DC1

This article cites 36 articles, 3 of which you can access for free at: http://n.neurology.org/content/91/4/e364.full\#ref-list-1

This article has been cited by 3 HighWire-hosted articles: http://n.neurology.org/content/91/4/e364.full\#\#otherarticles

This article, along with others on similar topics, appears in the following collection(s):

\section{All Pain}

http://n.neurology.org/cgi/collection/all_pain

Class I

http://n.neurology.org/cgi/collection/class_1

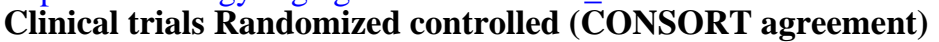

http://n.neurology.org/cgi/collection/clinical_trials_randomized_contro lled_consort_agreement

Migraine

http://n.neurology.org/cgi/collection/migraine

Vagus nerve stimulation

http://n.neurology.org/cgi/collection/vagus_nerve_stimulation

Information about reproducing this article in parts (figures,tables) or in its entirety can be found online at:

http://www.neurology.org/about/about_the_journal\#permissions

Information about ordering reprints can be found online:

http://n.neurology.org/subscribers/advertise

Neurology ${ }^{\circledR}$ is the official journal of the American Academy of Neurology. Published continuously since 1951, it is now a weekly with 48 issues per year. Copyright (C 2018 The Author(s). Published by Wolters Kluwer Health, Inc. on behalf of the American Academy of Neurology.. All rights reserved. Print ISSN: 0028-3878. Online ISSN: 1526-632X.

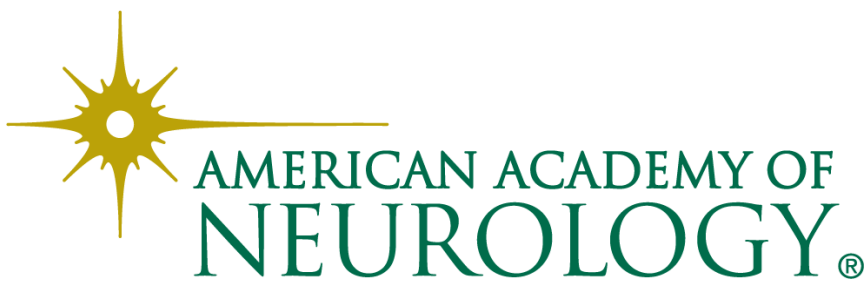

